\title{
Strangeness of the nucleon from lattice QCD
}

\author{
Simon Dinter ${ }^{a}$, Vincent Drach ${ }^{* a}$, Karl Jansen ${ }^{a}$ \\ ${ }^{a}$ NIC, DESY Zeuthen, Platanenallee 6, D-15738 Zeuthen, Germany \\ E-mail: vincent.drach@desy.de
}

We present lattice QCD results concerning the light and strange quark content of the Nucleon. The strangeness of the nucleon is an important source of uncertainty in the computation of the cross section between dark matter and ordinary nuclear matter. The direct computation of the corresponding matrix elements is known to be challenging, in particular because singlet quantities are very noisy on the lattice. Using an improved method, we obtain results in the light and strange sector that pave the way for a future analysis of the systematic effects. This proceedings contribution summarizes one of our recent works[1].

Sixth International Conference on Quarks and Nuclear Physics,

April 16-20, 2012

Ecole Polytechnique, Palaiseau, Paris

${ }^{*}$ Speaker. 


\section{Introduction}

The various evidences of the existence of dark matter have led to the development of experiments dedicated to detect dark matter directly. The detection relies on the measurements of the recoil of atoms hit by a dark matter candidate. One popular class of dark matter models involve an interaction between a WIMP and a Nucleon mediated by a Higgs exchange (see Fig. 1a for an illustration). Therefore, the scalar content of the nucleon is a fundamental ingredient in the WIMPNucleon cross section. In this way, the uncertainties of the scalar content translates directly into the accuracy of the constraints on beyond the standard model physics. Since the coupling of the Higgs to quarks is proportional to the quark masses, it is important to know how large scalar matrix elements of the nucleon are, in particular for the strange and charm quarks.

One common way to write the parameters entering the relevant cross section are the so-called sigma-terms of the nucleon:

$$
\sigma_{\pi N} \equiv m\langle N|\bar{u} u+\bar{d} d| N\rangle \quad \text { and } \quad \sigma_{0} \equiv m\langle N|\bar{u} u+\bar{d} d-2 \bar{s} s| N\rangle
$$

where $m$ denotes the light quark mass. Quantifying the scalar strangeness content of the nucleon a parameter $y_{N}$ is introduced,

$$
y_{N} \equiv \frac{2\langle N|\bar{s} s| N\rangle}{\langle N|\bar{u} u+\bar{d} d| N\rangle}
$$

which can be also related to the sigma terms of the nucleon in eq. (1.1).

A low energy theorem relates $\sigma_{\pi N}$ to the pion nucleon scattering amplitude extrapolated to the Cheng-Dashen point. The functional form of the extrapolation formula can be established using dispersion relation and in this way $\sigma_{\pi N}$ has been found to be $\sigma_{\pi N}=79(7) \mathrm{MeV}$ [2]. Note that a recent result which uses Lorentz covariant baryon chiral perturbation theory gives $\sigma_{\pi N}=$ 59(7) $\mathrm{MeV}[3]$. Concerning the $y_{N}$-parameter one finds for instance $y_{N}=0.44(13)$ [4]. As pointed out in ref. [5] and discussed above, it is quite important to provide a precise value for $\sigma_{\pi N}$ and $y_{N}$ in order to be able to interpret the ongoing and planned experimental searches of WIMPs. We refer for instance to [6] for more details and we illustrate this point by a result of the aforementionned paper in Fig. 1b where we show the elastic spin independent cross section Wimp-Nucleon for some SUSY benchmark models (referred by capital letters).

The direct computation of the above matrix elements is a technical challenge on the lattice for several reasons. First, it involves the computation of "singlet" or "disconnected" diagrams that are very noisy on the lattice. Second, discretisations that break chiral symmetry generally suffer from a mixing under renormalization between the light and strange sector, which is difficult to treat in a fully non-perturbative way. However, twisted mass fermions offer two advantages here: they provide both an efficient variance noise reduction for disconnected diagrams and a convenient way to avoid the chirally violating contributions that are responsible for the mixing under renormalization. We refer to [1] for detailled on the method and references.

In this contribution we argue that we developed an efficient method to perform an ab-initio computation of the sigma terms of the Nucleon. This open the doors of a - already ongoingdetailed study of the systematic effects, which is necessary in order to be able to provide reliable results for phenomenological analysis. 


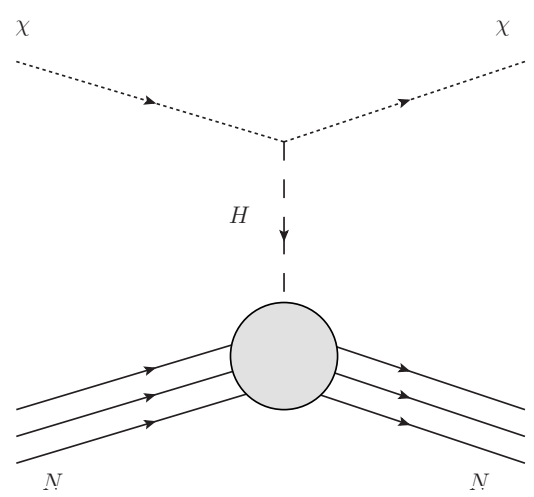

Figure 1: The Higgs-boson exchange contribution to the WIMP-Nucleon low energy scattering process

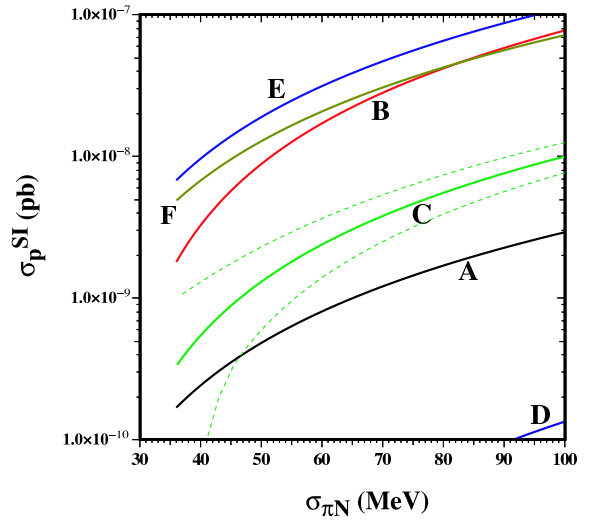

Figure 1: The Higgs-boson exchange contribution to the WIMP-Nucleon low energy scattering process (a) and example of the dependence on the $\pi$-Nucleon $\sigma$ term of the elastic spin independent cross section Wimp-Nucleon (b) for some SUSY benchmark models (referred by capital letters), taken from [6].

\section{Lattice techniques}

In our simulations we use the mass-degenerate twisted mass action in the light sector and the mass non-degenerate twisted mass action in the strange and charm sector. The quark masses of the heavy quark doublet have been tuned such that the Kaon and D-mesons masses assume approximately their physical value. The reader interested in more details about aspects of this setup is referred to $[7,8]$.

The twisted mass Dirac operator for a doublet of degenerate quarks reads :

$$
D_{\mathrm{tm}}[U]=D_{\mathrm{W}}[U]+m_{0}+i \mu_{q} \gamma_{5} \tau^{3}
$$

where $D_{\mathrm{W}}[U]$ is the massless Wilson-Dirac operator, $m_{0}$ the bare Wilson mass and $\mu_{q}$ the bare twisted mass parameter. Once the parameter $m_{0}$ is tuned to a situation referred to maximal twisted, the bare parameter $\mu_{q}$ is proportional to the mass of a doublet of degenerate quarks and all physical observables are free of discretization effects proportional to the lattice spacing (we refer to this property as automatic $O(a)$ improvement). This is one of the major advantages of this discretization. However this considerable advantage comes to the price that isospin and parity symmetries are broken at finite lattice spacing. Furthermore when considering the generalization of Eq. (2.1) to non-degenerate quarks, one has to break flavour conservation at finite lattice spacing. To avoid complication when considering the strange (or charm) quark content of the nucleon, we use a particular setup -called mixed action setup- in which we use a different action for the valence and for the sea quarks. The results obtained in both setup are however guaranteed to coincide once the continuum limit is taken. As mentionned earlier, we show in [1] that in this setup the sigma terms are renormalization free quantities.

In the following, $O_{q}=\bar{q} q$ with index $l, s, c$ will denote the relevant operator to compute the sigma terms in the light $(l)$, strange $(s)$ or charm $(c)$ sector.

Introducing the nucleon two-point function :

$$
C_{N, 2 \mathrm{pts}}^{ \pm}\left(t-t_{\mathrm{src}}, \vec{x}_{\mathrm{src}}\right)=\sum_{\vec{x}} \operatorname{tr} \Gamma^{ \pm}\left\langle\mathscr{J}_{N}(x) \overline{\mathscr{J}_{N}}\left(x_{\mathrm{src}}\right)\right\rangle
$$


where $x_{\text {src }} \equiv\left(t_{\text {src }}, \vec{x}_{\text {src }}\right)$ is the source point and the subscript $N$ refers to the proton or to the neutron states for which the interpolating fields are given by

$$
\mathscr{J}_{p}=\varepsilon^{a b c}\left(u^{a, T} \mathscr{C} \gamma_{5} d^{b}\right) u^{c} \quad \text { and } \quad \mathscr{J}_{n}=\varepsilon^{a b c}\left(d^{a, T} \mathscr{C} \gamma_{5} u^{b}\right) d^{c}
$$

The projectors used are $\Gamma^{ \pm}=\frac{1 \pm \gamma_{0}}{2}$, and $\mathscr{C}$ is the charge conjugation matrix. The nucleon threepoint functions is

$$
C_{N, 3 \mathrm{pts}}^{ \pm, O_{q}}\left(t_{s}, t_{\mathrm{op}}, \vec{x}_{\mathrm{src}}\right)=\sum_{\vec{x}, \vec{x}_{\mathrm{op}}} \operatorname{tr} \Gamma^{ \pm}\left\langle\mathscr{J}_{N}(x) O_{q}\left(x_{\mathrm{op}}\right) \overline{\mathscr{J}_{N}}\left(x_{\mathrm{src}}\right)\right\rangle
$$

The general form of the 3-point functions in Eq. (2.3) lead to both, connected ( illustrated in fig. 2a) and disconnected ( illustrated in 2 b) contributions. The desired scalar matrix elements can then be computed using the asymptotic behaviour of the ratio of a three and two-point functions:

$$
R_{O_{q}}\left(t_{s}, \Delta t_{\mathrm{op}}\right)=\frac{C_{N, 3 \mathrm{pts}}^{+, O_{q}, \mathrm{vev}}\left(t_{s}, t_{\mathrm{op}}\right)}{C_{N, 2 \mathrm{pts}}^{+}\left(t, x_{\mathrm{src}}\right)}=\langle N|\bar{q} q| N\rangle+\mathscr{O}\left(e^{-\Delta t_{\mathrm{op}}}\right)+\mathscr{O}\left(e^{-\Delta\left(t_{s}-t_{\mathrm{op}}\right)}\right) .
$$

where $\Delta$ stands for the difference of between the Nucleon and its first excited states.

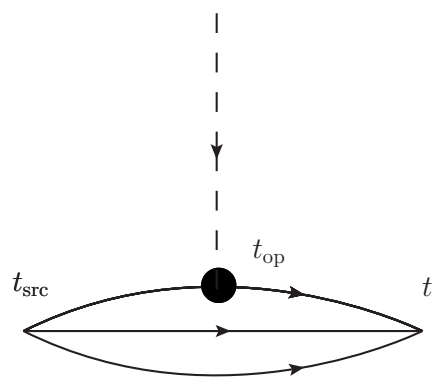

(a)

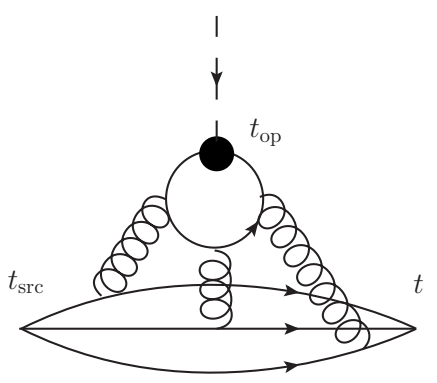

(b)

Figure 2: We illustrate the connected (left) and the disconnected (right) graphs that arise from the contractions leading to the 3-point function discussed in the text.

The disconnected contribution consists of a closed quark line connected to the valence quark line of the nucleon by an arbitrary number of gluons and is known to be extremely noisy in practice. In order to compute it, the basic problem is to estimate efficiently the trace of the inverse of the Dirac operator. Using a property of the twisted mass fermions, one can build an exact relation between the inverse of the two flavours Dirac operator and improve the performances compared to more standard approach using a fixed computational cost. This is illustrated in Fig. 3a where we show the signal over noise ratio at a fixed time $t_{s}$ and $\Delta t_{o p}$ for the method discussed in this contribution(black diamond) and another one (equivalent in terms of computer time) referred as hopping parameter expansion (orange dot). The twisted mass specific method clearly improved by a factor $\approx 3$ the signal-over-noise ratio for a fixed computing time.

\section{Results}

We first concentrate on the determination of $\sigma_{\pi N}$ defined in Eq. (1.1). As mentionned before there are two contributions to the ratio (2.4) referred as the connected and disconnected part. The 


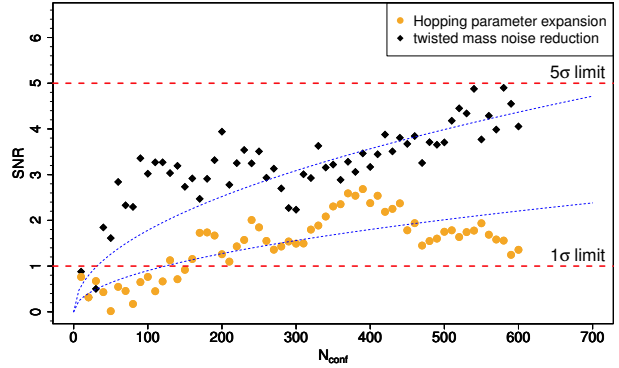

(a)

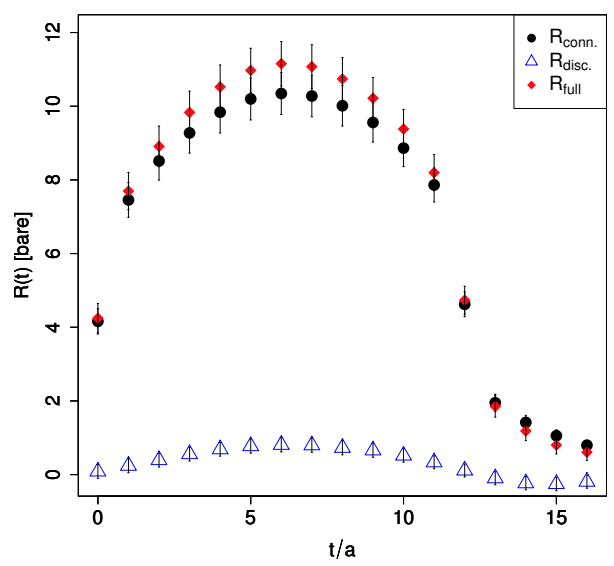

(b)

Figure 3: (a) Signal to noise ratio (SNR) of the quantity $R_{O_{s}}(12 a, 6 a)$, as a function of the number of gauge field configurations $N_{\text {conf }}$, for the variance noise reduction technique used in this paper and the hopping parameter expansion technique. The dashed curves are drawn to guide the eye. (b) Plot of the contributions

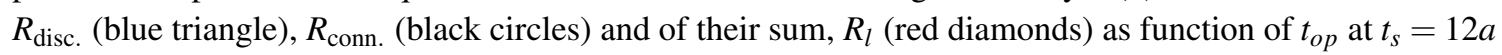

disconnected part is by far the most challenging one. Both the connected (black dot) the disconnected (blue triangle) and their sum are represented in in Fig. 3b. As can be seen a clear signal is obtained. The disconnected contribution is small compare to the connected one. It is important to recall that such a "plateau" allows to extract the value of $\sigma_{\pi N}$ obtained at a pion mass of about $380 \mathrm{MeV}$ in a fixed volume and at finite lattice spacing. Maybe even more important are the corrections due to the fact that $t_{s}$ and $t_{o p}$ are not sent to infinity and thus lead to the so-called excited states contamination.

We perform the same analysis in the strange sector (see Fig. 4), in which there is no connected contribution to the ratio (2.4) because the nucleon does not contain strange quark in the valence. Here we conclude that a plateau can be found which is $\approx 5 \sigma$ different from zero. Again, one has to investigate systematic effects before being able to claim that this can be used in a phenomenological study.

Combining this value with the result for the scalar matrix element obtained in the light quark sector discussed above, allows us finally to compute $y_{N}$. We find a small value in line with what is found by other lattice collaboration, namely $y_{N}=0.082(16)$. The value we obtain, ignoring most of the systematic effects, is small compared to estimates from chiral perturbation theory but is in line with recent lattice results obtained by other groups.

We also carried out the first feasibility study of the charm quark content of the nucleon. This is possible because we have at our disposal $N_{f}=2+1+1$ simulations with a fully dynamical charm quark degree of freedom. We show in Fig. 5 the dependence of $R_{c}$ on $t_{o p}$ (at $t_{s}=12 a$ ), using exactly the same statistics as in the light and in strange quark sectors. Unfortunately, for the charm quark content no hint of a plateau is visible. Signal and noise have equal order of magnitude and our results are compatible with zero. For comparison we also show the results for the strange quark content obtained in the previous section as a grey band. From our data we can only establish the inequality $\left|\left\langle N\left|O_{c}\right| N\right\rangle\right| \lesssim\left|\left\langle N\left|O_{s}\right| N\right\rangle\right|$. 


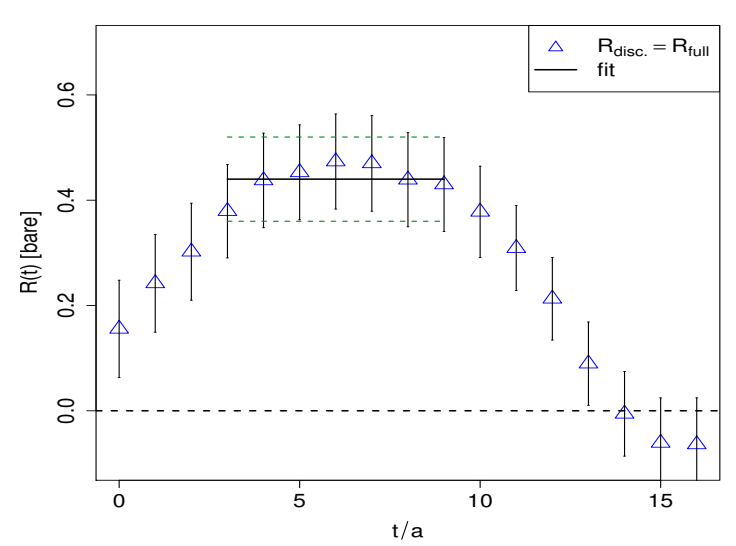

Figure 4: Time dependence of $R_{\text {disc. }}$ in the strange quark mass regime $\left(a \mu_{q}=0.018\right)$. The source-sink separation has been fixed to $12 a$ and 842 configurations have been used.

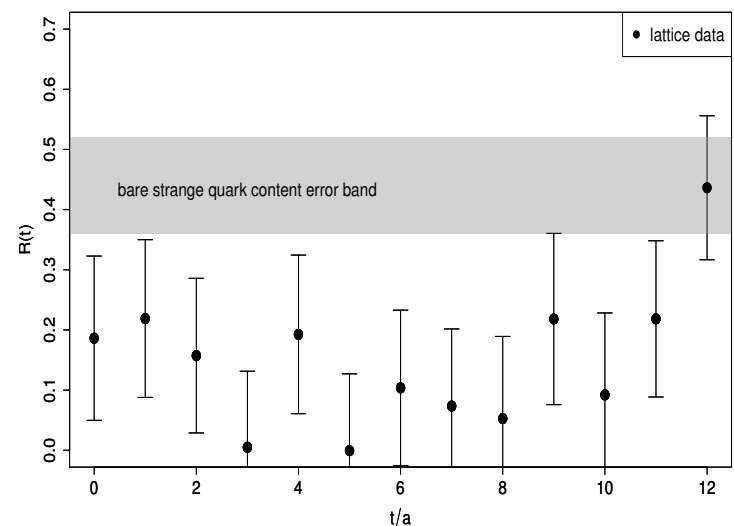

Figure 5: The ratio $R_{c}$ for fixed $t_{s}=12 a$ versus $t_{\mathrm{op}}$ in the charm quark mass regime $\left(a \mu_{c}=0.2\right)$. For comparison, we display as a grey band the plateau value obtained in the strange quark case

\section{Conclusion}

In this proceedings contribution we present a method that uses properties of the twisted mass action to improve the statistical accuracy of quantities such that the sigma terms of the nucleon. This technical step is necessary to be able to improve our knowledge of the systematic effects such that the excited states contamination, the volume or the discretization effects. Once they will be under control one could perform extrapolation to the physical quark masses and obtain a reliable results relevant for phenomenological studies.

\section{Acknowledgments}

This work was performed using HPC resources provided by the JSC Forschungszentrum Jülich on the JuGene supercomputer and by GENCI (IDRIS-CINES) Grant 2010-052. It is supported in part by the DFG Sonderforschungsbereich/ Transregio SFB/TR9.

\section{References}

[1] S. Dinter, V. Drach, R. Frezzotti, G. Herdoiza, K. Jansen and G. Rossi, arXiv:1202.1480 [hep-lat].

[2] M. M. Pavan, I. I. Strakovsky, R. L. Workman and R. A. Arndt, PiN Newslett. 16, 110 (2002) [hep-ph/0111066].

[3] J. M. Alarcon, J. Martin Camalich and J. A. Oller, Phys. Rev. D 85, 051503 (2012) [arXiv:1110.3797 [hep-ph]].

[4] R. D. Young and A. W. Thomas, Nucl. Phys. A 844, 266C (2010) [arXiv:0911.1757 [hep-lat]].

[5] J. Giedt, A. W. Thomas and R. D. Young, Phys. Rev. Lett. 103, 201802 (2009) [arXiv:0907.4177 [hep-ph]].

[6] J. R. Ellis, K. A. Olive, Y. Santoso and V. C. Spanos, Phys. Rev. D 71, 095007 (2005) [hep-ph/0502001].

[7] R. Baron, P. .Boucaud, J. Carbonell, A. Deuzeman, V. Drach, F. Farchioni, V. Gimenez, G. Herdoiza et al., JHEP 1006, 111 (2010). [arXiv:1004.5284 [hep-lat]].

[8] R. Baron et al. [ European Twisted Mass Collaboration ], Comput. Phys. Commun. 182, 299-316 (2011). [arXiv:1005.2042 [hep-lat]]. 